\title{
Modeling and Simulation of Electroactive Polymer Robotic Actuator
}

\author{
Md. Masum Billah, Raisuddin Khan and Amir Akramin Shafie \\ Department of Mechatronics Engineering, Faculty of Engineering \\ International Islamic University Malaysia \\ 53100 Kuala Lumpur, Malaysia \\ mdmasum.b@live.iium.edu.my
}

\begin{abstract}
Flexible snake robotic actuator inspired by the performance of snakes together with muscular and vertebrate, well in obstacle constrained rough environment and are capable of complex motions. These types of actuator possess a wide range of motion while also achieving complex geometrical configurations. Although, flexible structures that mimic muscular actuation like the snake links have been attempted in the literature by using shape memory alloys (SMAs), or strings and cables, light-weight, relatively powerdense dielectric electroactive polymers (EAP) can also be used in unison with a flexible snake robot actuator structure to provide actuation. This paper presents a theoretical and experimental study of the Dielectric EAP (DEAP) actuator for designing flexible snake robot actuator. DEAP is silicon artificial muscles that will actuated by applying voltage which perform a great actuation due to its linear large deflection. Preliminary efforts have been made to develop a prototype actuator design as well as learn about the EAP material properties through experimentation.
\end{abstract}

Keywords-electroactive polymer; robot; actuator; muscle.

\section{INTRODUCTION}

Snake robots are of special interest to both robotics engineers and materials scientists because they combine extreme flexibility (they can bend at any point) with a capability for executing various sophisticated tasks, such as small hole navigation, manipulating small objects etc $[1,2]$. Engineered flexible structures that mimic muscular robots have been attempted in the literature, an actuator was demonstrated [3,4], by using pneumatic air muscles (PAM), which exhibits disadvantages of less precision, actuation delays, and a bulky compressed air generator. Also, PAM-based actuators cannot be used for light-weight robots. In essence, artificial muscles can rival biological counterparts have yet to be reported.

In an attempt to mimic the functionality of a muscular body by achieving similar high local curvature and complex configurations, different designs for a articulated snake robot actuator design that utilizes dielectric electro-active polymers (EAPs) as the artificial muscles have been explored. Compare with other materials used in the literature, EAPs are the only viable option to build a lightweight actuator for highly compliant artificial muscles. Polymer-based actuator materials are expected to work better than piezoelectric ceramic (PZT) actuators and shape-memory alloys (SMAs) in terms of the amount of strain achieved during actuation (0.1\% for PZTs [5]) and the electromechanical coupling that limits the large-scale maneuverability respectively [6]. They can also provide power densities within a factor of 3-5 of the electric motors but with the added flexibility of their muscle-like nature. Furthermore,

This research is supported by Science grant, Ministry of Science, Technology and Innovation (MOSTI) Malaysia. dielectric EAPs are good candidates for actuation because they exhibit quick response times and are capable of relatively large strains (10-100\%). DEAPs are commercially available in the form of ribbons and sheets, and are typically prestrained on compliant frames (up to 500\%) to reduce the actuation voltage. Prestraining is the act of stretching the elastomer, in either a unilateral direction or bilaterally, to reduce the thickness of the material.

Significant work has been done to explore the potential of the EAPs in actuator technologies while attempting to model the behaviour of this material. Out-of-plane actuation has been looked at and implemented using an agonist-antagonist configuration of the EAPs [7], and finite element methods (FEM), as well as analytical modelling of a circular in-plane EAP actuator has been examined [8]. The viscoelastic properties of VHB4910, a commercially available dielectric EAP, have also been characterized in the modelling process [9]. Different actuation methods like the spring roll actuator, and the contractive EAP actuator have been designed, developed, and manufactured to show their effectiveness $[9,10,11]$. Although there is extensive prior work in developing and modelling actuators separately, few works have been done to consolidate this technical know-how to design a truly flexible and complaint robotic actuator that resembles an flexible snake robot.

In this paper, preliminary steps have been taken in this direction of a design concept for this type of flexible actuator while utilizing a hyper-elastic material model to assist in the design process. 


\section{EAP BACKGROUND}

A number of conventional actuator technologies are being used today which include hydraulic, pneumatic, and electromechanical actuators. However, one common drawback that conventional actuators have is that the actuator itself or the power source is bulky and heavy. The need for light-weight, compliant, small-sized actuators yet having high power to weight ratio has driven research into active materials. Dielectric EAP consist of a thin film of elastomer sandwiched between to compliant electrodes and essentially are compliant capacitors that actuate when a DC voltage potential shown in Fig. 1 is applied across the electrodes. The electrodes have opposite charges hence attracted each other and thereby squeezing the thin film of elastomer. Since the elastomer is incompressible, it expands in the planar direction and thins in the thickness direction. Also, the same charges on an electrode repel each other leading to further expansion in the planar direction. This squeezing stress is called a compressive Maxwell stress. The areas that are covered in electrodes are called the active zones. This actuation strain is the source of motion for any actuator made from EAPs. However, most actuators must also utilize the prestrain of the EAP to allow for more efficient use of the motion. Generally, the larger the prestrain is, the lower the voltage required to activate the EAP. The tension-compression type of actuation can be seen from various actuators like a push-pull actuator, and a hinge actuator [7].
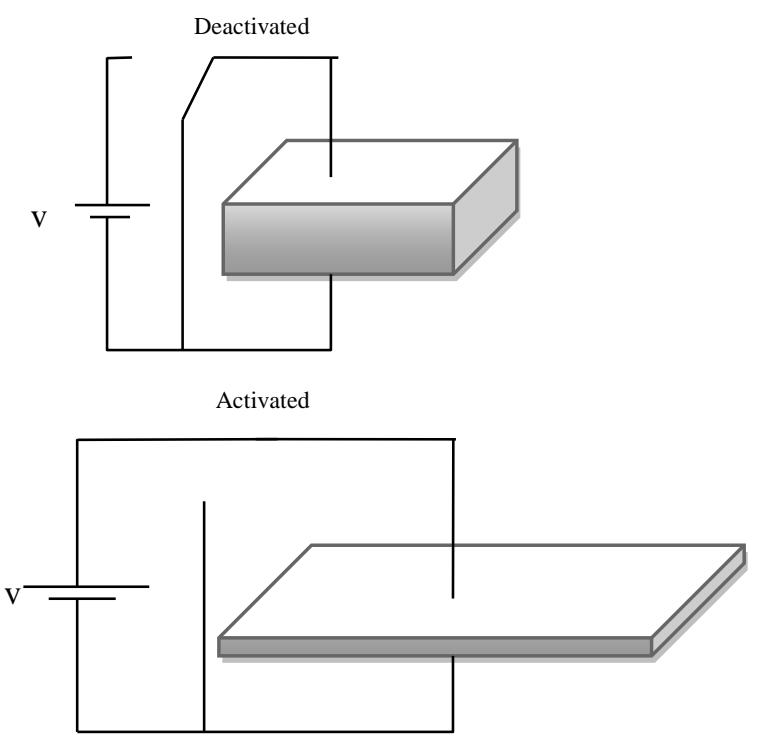

Fig. 1. Dielectric EAP actuator in deactivated (left) and activated state (right).

The previously mentioned actuators all use the extension of the EAPs to provide actuation; however, some actuators are designed to take advantage of the reduction in thickness of the EAP to provide actuation. These actuators require multiple layers of the elastomer to visibly observe the contraction. The active zones alternate in polarity for each layer so that each layer of the actuator is squeezed. Widely-used dielectric elastomers (DEs) are made out of either silicone or acrylic. VHB 4910 is acrylic EAPs that can strain more and are capable of more tensile stress during Prestraining (increased elastic energy density) when compared to silicone EAPs, however, silicone EAPs have a faster response time and are more efficient mechanically [12].

\section{ANALYSIS OF DEAP}

\section{A. Constitutive Models}

The purpose of the tensile tests were to determine the material parameters of the VHB 4910 DEAP that would be used in the modelling the behaviour of the material and will provide vital information on designing and predicting the movement of specific snake robot link designs. These material parameters are constants in the strain energy function denoted by $\mathrm{W}$. The general form of the strain energy function of a hyper-elastic material subject to a axial stress is denoted by equation (1) [13].

$$
\begin{aligned}
& \mathrm{W}=\sum_{\mathrm{a}=0}^{3} \sum_{\mathrm{b}=0}^{3} \mathrm{C}_{\mathrm{ab}}\left(\mathrm{I}_{1}-3\right)^{\mathrm{a}}\left(\mathrm{I}_{2}-3\right)^{\mathrm{b}} \\
& \mathrm{I}_{1}=\lambda_{1}^{2}+\lambda_{2}^{2}+\lambda_{3}^{2} \\
& \mathrm{I}_{2}=\lambda_{1}^{2} \lambda_{2}^{2}+\lambda_{2}^{2} \lambda_{3}^{2}+\lambda_{1}^{2} \lambda_{3}^{2}
\end{aligned}
$$

$I_{1}$ and $I_{2}$ denote the first and second invariant of the socalled left Cauchy-Green stress tensor. $\lambda_{i}(i=1 ; 2 ; 3)$ are the principal strain ratios. Note that I1 and I 2 are functions of the principal strain ratios. 1 ; 2 ; or 3 denotes the direction on the local elastomer sample in which the strains and stresses act. 1 denotes direction in which the tensile stress for the uniaxial test acts, 2 is the direction perpendicular to 1 in the plane of the material, and 3 is the direction resulting from the cross product of 1 and 2 is shown in Fig. 2. Cabs are the material parameters in which the uniaxial tests were performed to determine. Based on the strain-energy function, equations (3) and (4) are used for an incompressible, hyperelastic material to determine the principal stresses in terms of the principal strain ratios.

$$
\begin{aligned}
& \sigma_{11}=\lambda_{1}^{2} \frac{\partial \mathrm{W}}{\partial \mathrm{I}_{1}}+\lambda_{1}^{2}\left(\lambda_{2}^{2}+\lambda_{3}^{2}\right) \frac{\partial \mathrm{W}}{\partial \mathrm{I}_{2}}+\text { Phydro } \\
& \sigma_{22}=\lambda_{2}^{2} \frac{\partial \mathrm{W}}{\partial \mathrm{I}_{1}}+\lambda_{2}^{2}\left(\lambda_{3}^{2}+\lambda_{1}^{2}\right) \frac{\partial \mathrm{W}}{\partial \mathrm{I}_{2}}+\text { Phydro } \\
& \sigma_{33}=\lambda_{3}^{2} \frac{\partial \mathrm{W}}{\partial \mathrm{I}_{1}}+\lambda_{3}^{2}\left(\lambda_{1}^{2}+\lambda_{2}^{2}\right) \frac{\partial \mathrm{W}}{\partial \mathrm{I}_{2}}+\text { Phydro } \\
& \lambda_{1} \lambda_{2} \lambda_{3}=1
\end{aligned}
$$




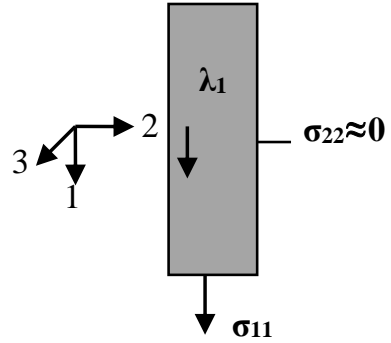

$a$

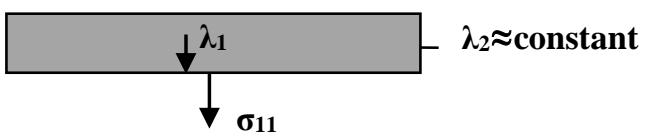

$b$

Fig. 2. Elastromer sample (a) Long and thin, (b) Wide-strip

$\sigma_{11}, \sigma_{22}, \sigma_{33}$ denotes the normal stress acting on the material in the 1,2 , and 3 directions and Phydro is the hydrostatic pressure. Equation (7) is the incompressible material condition which can be assumed for the VHB4910 elastomer. For boundary conditions specific to the uniaxial test, the experimental data was fitted to the first equation (4) in the 1 direction. For instance, for a long, thin elastomer strip, the boundary conditions were $\sigma_{22}=0, \sigma_{33}=0$. Figure 1 (a) shows what a uniaxial test with a long, thin elastomer sample would look like.

Long, thin elastomer samples are samples in which the original, unprestrained length is much greater than the width. This was done to reduce the amount of necking in the sample so that the assumptions for the boundary conditions of the simulation would be a good approximation (i.e. $\sigma_{22}=0$ ). Widestrip elastomer samples are samples in which the original, unprestrained width is much greater than the length. The assumptions for the boundary conditions of this sample are that the prestrain in the 2 direction remains constant ( $\lambda_{2}=$ const) and $\sigma_{33}=0$. Based on the correct boundary conditions, the stressto-strain relationship was derived using equations (7) and (8) and simplified to equations (5).

$$
\begin{gathered}
\sigma_{11}=\left(\lambda_{1}^{2}-\frac{1}{\lambda_{1}^{2} \lambda_{2}^{2}}\right) \frac{\partial \mathrm{W}}{\partial \mathrm{I}_{1}}+\left(-\frac{1}{\lambda_{1}^{2}}+\lambda_{1}^{2} \lambda_{2}^{2}\right) \frac{\partial \mathrm{W}}{\partial \mathrm{I}_{2}} \\
\sigma_{22}=\left(\lambda_{2}^{2}-\frac{1}{\lambda_{1}^{2} \lambda_{2}^{2}}\right) \frac{\partial \mathrm{W}}{\partial \mathrm{I}_{1}}+\left(-\frac{1}{\lambda_{2}^{2}}+\lambda_{1}^{2} \lambda_{2}^{2}\right) \frac{\partial \mathrm{W}}{\partial \mathrm{I}_{2}}
\end{gathered}
$$

Equation (5) is used to analyze both the long, thin elastomer and wide-strip elastomer samples, however, the correct boundary conditions need to be applied. For instance, when determining $\sigma_{11}$ in terms of $\lambda_{1}$ for a long, thin elastomer sample, $\sigma_{22}$ was set to zero and $\lambda_{2}$ was solved for to get an expression in terms of $\lambda_{1}$. This was plugged back into the $\sigma_{22}$ equation to get an expression only in terms of $\lambda_{1}$. For analyzing a wide-strip sample, the $\sigma_{22}$ equation was ignored because the $\sigma_{11}$ equation is already in terms of $\lambda_{1}$. $\lambda_{2}$ was simply the prestrain of the sample in the 2 direction since it was assumed to be constant. Both types of samples were tested and simulated. Once the experimental data was collected, the data was fitted to a stress-strain model and the material parameters were determined. Then, simulations using these material parameters were done to examine the disparity between the simulation results and the experimental data.

\section{B. Theoretical Analysis of Linear EAP Actuators}

In the designs of snake robot actuator, a linear EAP actuator is utilized or the actuation can be approximated by a linear EAP actuator. This means that upon activation of the EAP, the resulting strain that occurs is dominated in one direction (denoted by the 1 direction). Equations (11) show the constitutive model of the linear EAP actuator and were used to draw some insight on the design of an actuator.

$$
\begin{array}{r}
\sigma_{11}=\lambda_{1}^{2} \frac{\partial \mathrm{W}}{\partial \mathrm{I}_{1}}+\lambda_{1}^{2}\left(\lambda_{2}^{2}+\frac{1}{\lambda_{1}^{2} \lambda_{2}^{2}}\right) \frac{\partial \mathrm{W}}{\partial \mathrm{I}_{2}}+\text { Phydro }=\frac{\mathrm{F}_{1}}{\mathrm{y}^{\mathrm{I}} \lambda_{3} \mathrm{z}_{0}} \quad(10) \\
\sigma_{22}=\lambda_{1}^{2} \frac{\partial \mathrm{W}}{\partial \mathrm{I}_{1}}+\lambda_{3}^{2} \frac{\partial \mathrm{W}}{\partial \mathrm{I}_{1}}+\lambda_{2}^{2}\left(\lambda_{1}^{2}+\frac{1}{\lambda_{1}^{2} \lambda_{2}^{2}}\right) \frac{\partial \mathrm{W}}{\partial \mathrm{I}_{2}}+\text { Phydro }(11) \\
\sigma_{33}=\frac{1}{\lambda_{1}^{2} \lambda_{2}^{2}} \frac{\partial \mathrm{W}}{\partial \mathrm{I}_{1}}+\frac{1}{\lambda_{1}^{2} \lambda_{2}^{2}}\left(\lambda_{1}^{2}+\lambda_{1}^{2}\right) \frac{\partial \mathrm{W}}{\partial \mathrm{I}_{2}}+\text { Phydro }= \\
\frac{\epsilon_{\mathrm{r}} \epsilon_{0} \mathrm{~V}^{2}}{\left(\lambda_{3} \mathrm{z}_{0}\right)^{2}}
\end{array}
$$

This model includes the compressive stress that effectively "squeezed" the EAP together in the thickness direction which is denoted by the right hand side of the third equation. yI is the width of the actuator after prestrain, $\epsilon_{\mathrm{r}}$ is the free-space dielectric permittivity $\left(\epsilon_{\mathrm{r}}=8.8510 .12 \mathrm{~F} / \mathrm{m}\right), \epsilon_{0}$ is the relative permittivity of the dielectric material $\left(\epsilon_{0}=4.7\right.$ for VHB 4910 [14]), $\mathrm{V}$ is the voltage difference supplied to the electrodes, and $\mathrm{z}_{0}$ is the original thickness of the actuator when it is not activated. For some linear actuator designs, there may be free edges such that the actuator is not attached to a rigid structure at every edge. This causes necking and, as a result, the width of the actuator varies along the actuation direction. Solving for the hydrostatic pressure, the following equation is obtained. 


$$
\text { Phydro }=\frac{\epsilon_{\mathrm{r}} \epsilon_{0} \lambda_{1}^{2} \lambda_{2}^{2} \mathrm{~V}^{2}}{\mathrm{z}_{0}^{2}}-\frac{1}{\lambda_{1}^{2} \lambda_{2}^{2}} \frac{\partial \mathrm{W}}{\partial \mathrm{I}_{1}}+\frac{1}{\lambda_{1}^{2} \lambda_{2}^{2}}\left(\lambda_{1}^{2}+\lambda_{2}^{2}\right) \frac{\partial \mathrm{W}}{\partial \mathrm{I}_{2}}
$$

This expression is substituted back into the first equation to yield the following:

$$
\begin{array}{r}
\mathrm{F}_{1}=\frac{\mathrm{y}^{\mathrm{I}} \mathrm{z}_{0}}{\lambda_{1} \lambda_{2}}\left[\lambda_{1}^{2} \frac{\partial \mathrm{W}}{\partial \mathrm{I}_{1}}+\lambda_{1}^{2}\left(\lambda_{2}^{2}+\frac{1}{\lambda_{1}^{2} \lambda_{2}^{2}}\right) \frac{\partial \mathrm{W}}{\partial \mathrm{I}_{2}}-\frac{\epsilon_{\mathrm{r}} \epsilon_{0} \lambda_{1}^{2} \lambda_{2}^{2} \mathrm{~V}^{2}}{\mathrm{z}_{0}^{2}}-\right. \\
\left.\frac{1}{\lambda_{1}^{2} \lambda_{2}^{2}} \frac{\partial \mathrm{W}}{\partial \mathrm{I}_{1}}-\frac{1}{\lambda_{1}^{2} \lambda_{2}^{2}}\left(\lambda_{1}^{2}+\lambda_{2}^{2}\right) \frac{\partial \mathrm{W}}{\partial \mathrm{I}_{2}}\right] \\
\sigma_{11}=\lambda_{1}^{2} \frac{\partial \mathrm{W}}{\partial \mathrm{I}_{1}}+\lambda_{1}^{2}\left(\lambda_{2}^{2}+\frac{1}{\lambda_{1}^{2} \lambda_{2}^{2}}\right) \frac{\partial \mathrm{W}}{\partial \mathrm{I}_{2}}-\frac{\epsilon_{\mathrm{r}} \epsilon_{0} \lambda_{1}^{2} \lambda_{2}^{2} \mathrm{~V}^{2}}{\mathrm{z}_{0}^{2}}-\frac{1}{\lambda_{1}^{2} \lambda_{2}^{2}} \frac{\partial \mathrm{W}}{\partial \mathrm{I}_{1}}- \\
\frac{1}{\lambda_{1}^{2} \lambda_{2}^{2}}\left(\lambda_{1}^{2}+\lambda_{2}^{2}\right) \frac{\partial \mathrm{W}}{\partial \mathrm{I}_{2}}(15)
\end{array}
$$

Since the stress invariants can be written in terms of just $\lambda_{1}$ and $\lambda_{2}$ from the incompressible assumption, the force of the linear actuator in the 1 direction (direction of dominate strain) is only a function of $\mathrm{y}^{\mathrm{I}}, \mathrm{z}_{0}, \lambda_{1}$ and $\lambda_{2}$ for a given material and a given activation voltage $\left(\mathrm{F}_{1}=\mathrm{f}\left(\mathrm{y}^{\mathrm{I}}, \mathrm{z}_{0}, \lambda_{1}, \lambda_{2}\right)\right)$. Equation (14) also tells that the force of the EAP actuator will vary linearly with the width and the thickness of the actuator. Also, the width of the actuator is determined by the prestrain in that direction so the force is also implicitly dependent in the prestrain in this manner. This physically means that the width, thickness, and prestrain of the actuator determines how much force is available for the arm to utilized, but the length of the actuator (dimension of the actuator in the activation direction) has no effect. However, if the linear actuator has free edges, then the length will have an effect on the force of the actuator. For instance, longer actuators for a given width would have a less significant necking effect. The necking of the free edges effectively decrease the prestrain of the actuator in that direction and since the force is a function of prestrain, the force would change with different degrees of necking.

Examining equation (14), the stress in the activation direction is solely a function of prestrain given an activation voltage and thickness. It is not a function of the size of the actuator therefore; theoretically, a large EAP actuator and a small EAP actuator, regardless of the width to length ratio, with equal prestrain and thickness should have the same internal stresses during activation or inactivation. However, for linear actuators with free edges and using the same analysis as previously mentioned, the internal stresses would be different for a longer actuator (for a given width) because of a decreased necking effect. This leads to the conclusion that only linear EAP actuators subject to free edges with the same width-tolength ratio and same prestrain can have equal internal stresses given a specific strain. Furthermore, this leads to the fact that the coefficients of the strain energy function, also known as the material parameters of the material, should be the same for these actuators. As a consequence, determining these material parameters with uniaxial tests will allow for stress and strain prediction of different sized but similar-shaped EAP actuators.

\section{Theoretical Data Analysis for Long, Thin Elastomer Sample}

First, uniaxial tensile simulation were done, thin strip of elastomer (refer to Fig. 1 (up)). From the gathered data points, the deflection measurements and loads needed to be converted into principal strains and stresses in order for the first equation in (8) to be fitted and the material parameters to be determined. So, for the experimental data analysis, the principal stress in the 1 direction was calculated by equation (9).

$$
\sigma_{11}=\frac{P}{A}
$$

A denotes the cross sectional area of the sample in the nonnecking region, and $\mathrm{P}$ denotes the amount of force acting on the sample in the elongation direction calculated by $\mathrm{P}=\mathrm{mg}$. The cross sectional area was calculated by multiplying the measured width of the non-necking region and the thickness. The thickness was calculated using the incompressible assumption equation (7). The principal strain $\left(\lambda_{1}\right)$ is equal to $\frac{x}{x_{0}}$ where $\mathrm{x}$ is the instantaneous length of the elastomer sample in the 1 direction and $x_{0}$ is the initial length of the elastomer in the elongation direction corresponding to the zero prestrain.

The theoretical data was converted from force to stress and from deflection to strain, Matlab's curve-fitting toolbox was used to determine the material parameters corresponding to the first equation in (8). For one experiment with initial prestrain of $\lambda_{1}=1: 1389, \lambda_{2}=1$ and an initial length in the 1 direction of $6.75 \mathrm{~mm}$, the material parameters for the strain energy model were determined and are shown in Table 1.

The simulation process is done with the boundary conditions $\left(\sigma_{22}=\sigma_{33}=0\right)$ for long, thin elastomer strip samples. The superscript I refers to the pre-strained configuration whereas the II refers to the loaded configuration. $\mathrm{y}^{\mathrm{I}}$ is the width (in direction 2). $\mathrm{z}_{0}$ is the original thickness of the elastomer sample prior to prestrain which is $1 \mathrm{~mm}$. This simulation process was performed for loads ranging from the minimum to maximum load. The nominal strain was calculated using equation (17).

$$
\mathrm{s}=\left(\frac{\lambda_{1}^{\mathrm{II}}}{\lambda_{1}^{\mathrm{I}}}-1\right) 100 \%
$$

TABLE I. MATERIAL PARAMETERS FOR A LONG, THIN ELASTROMER SAMPLE

\begin{tabular}{|c|c|}
\hline Material Parameter & Fitted Value (Pa) \\
\hline C01 & -9.215 \\
\hline C02 & 274.9 \\
\hline C03 & -1.264 \\
\hline C10 & -6.944 \\
\hline C11 & -50.02 \\
\hline C12 & 267.6 \\
\hline
\end{tabular}


ICIT 2015 The $7^{\text {th }}$ International Conference on Information Technology

doi:10.15849/icit.2015.0091 C ICIT 2015 (http://icit.zuj.edu.jo/ICIT15)

\begin{tabular}{|c|c|}
\hline $\mathrm{C} 13$ & 0.4922 \\
\hline $\mathrm{C} 20$ & 165.9 \\
\hline $\mathrm{C} 21$ & 0.432 \\
\hline C22 & -19.28 \\
\hline C23 & -0.01165 \\
\hline C30 & 300.1 \\
\hline C31 & -0.8353 \\
\hline C32 & 0.2947 \\
\hline C33 & $6.147 \mathrm{e}-1$ \\
\hline
\end{tabular}

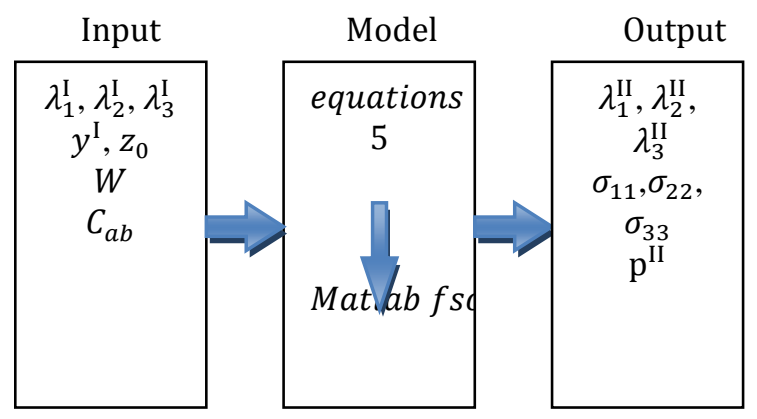

Fig. 3. Simulation process

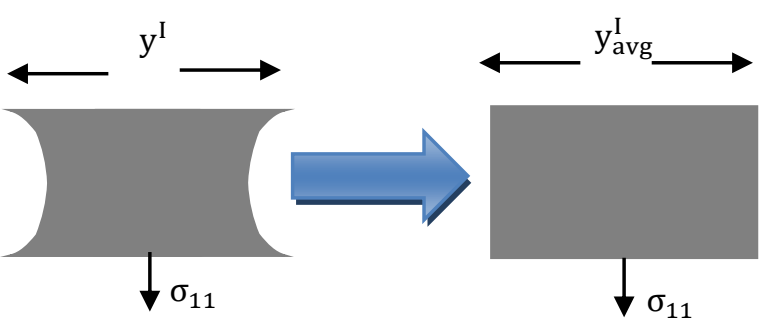

each data point. An average width would then be calculated from that parabolic width distribution and this width would be assumed to be constant along the elongation direction of the elastomer sample. This product of the average width and the corresponding thickness calculated by the incompressible assumption equation (4) was the approximated cross sectional area. Fig. 4 shows this approximation. $y_{\text {avg }}^{I}$ would be less than $\mathrm{y}^{\mathrm{I}}$. This approximation was then made for each data point and the $y_{\text {avg }}^{\mathrm{I}}$ for each data point was different because the minimum width was different for each data point. The principal stress was then calculated by equation (14). The principal strains $\left(\lambda_{1}\right)$ were calculated by $\frac{x}{x_{0}}$ where $x$ is the current length of the elastomer sample in the elongation direction and $x_{0}$ is the initial length of the elastomer corresponding to the zero prestrain. Table 2 shows the material parameters for a widestrip elastomer samples with initial lengths of $21.7 \mathrm{~mm}, 25.4$ $\mathrm{mm}, 33.8$, and widths of $63.5 \mathrm{~mm}, 76.2 \mathrm{~mm}$, and $101.6 \mathrm{~mm}$ respectively. The samples were subject to an initial prestrain of $\lambda_{1}=3, \lambda_{2}=5$ ( 3 in the actuation direction) with an equal width-to-length ratio of about 3 . It was evident from the uniaxial tests that the stress-strain curves for all three samples are similar and warrants the fact that the material parameters are the same for different sized actuators with equal width-tolength ratios and equal prestrains (given the error in measurement). The 3 by 5 prestrain of the sample was chosen as it is the prestrain configuration that has shown the best stress and strain properties in terms of using the elastomer as a linear actuator [8]. The material parameters were determined by fitting experimental data to the first equation in (8).

TABLE II. MATERIAL PARAMETERS FOR A WIDE STRIP ELASTROMER SAMPLE

\begin{tabular}{|c|c|}
\hline Material Parameter & Fitted Value (Pa) \\
\hline $\mathrm{C} 01$ & 1.2738 \\
\hline $\mathrm{C} 02$ & 2.1262 \\
\hline $\mathrm{C03}$ & 0.8228 \\
\hline $\mathrm{C} 10$ & -3.774 \\
\hline $\mathrm{C} 11$ & 1.5459 \\
\hline $\mathrm{C} 12$ & -4.1087 \\
\hline $\mathrm{C} 13$ & 0.0033 \\
\hline $\mathrm{C} 20$ & 8.1668 \\
\hline $\mathrm{C} 21$ & -2.7571 \\
\hline $\mathrm{C} 22$ & -14.3687 \\
\hline $\mathrm{C} 23$ & -0.0264 \\
\hline $\mathrm{C} 30$ & 0.2192 \\
\hline C31 & 1.1898 \\
\hline $\mathrm{C} 32$ & 0.6432 \\
\hline C33 & $1.0505 \mathrm{e}-5$ \\
\hline
\end{tabular}

\section{Theoretical Data Analysis for Wide-Strip Elastomer Samples}

The wide-strip elastomer sample was analyzed differently from the long, thin elastomer because the boundary conditions are different. The pre-strained length of the wide-strip elastomer sample was much smaller than the pre-strained width. On the other hand, the pre-strained length of the long, thin elastomer was much greater than the pre-strained width. This was the fundamental difference. It was a good approximation in the long, thin elastomer case to assume that the stress in the 2 direction $\left(\sigma_{22}\right)$ was negligible and can be set to zero. This was not a good assumption in this case. However, assuming that the principal strain in the 2 direction $\left(\lambda_{2}\right)$ was constant turned out to be a much more reasonable assumption. Therefore, only the first equation in equation (8) was needed. To determine the principal stresses and strains from the experimental data, some approximations were made. The necking was approximated geometrically by a parabolic shape determined by the minimum width of the elastomer sample at 


\section{RESULTS AND DISCUSSIONS}

The design of dielectric elastomer is such a way that it can perform large variety of motions depends on the different arrangements of dielectric elastomer strip. We proposed a novel design of elongating elastomer ribbon that will be used as the tendon. The model exhibits linear motion along the axis. The elongating characteristics appear once the dielectric elastomer is electrically activated. The VHB 4910 transparent polymer from $3 \mathrm{M}$, an available EAP for commercial purpose, is found in the market. The ribbon of DE tendons is considered $30 \mathrm{~mm}$ long in length. The stress-strain investigation of the material is shown in Fig. 5 (a), (b).

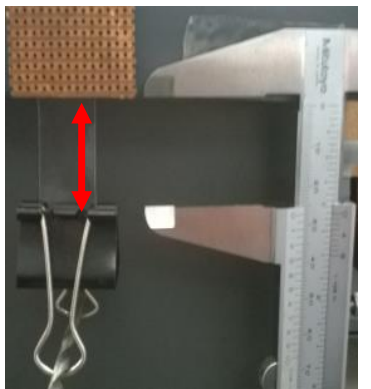

(a)

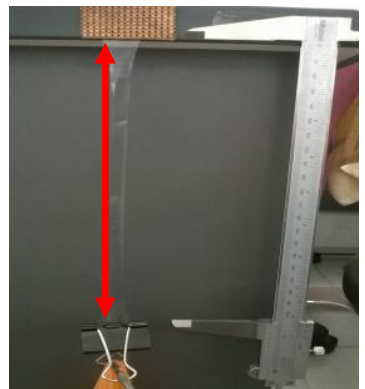

(b)
Fig. 5. Elongation test for VHB4910 ribbon: (a) initial length without (b) final length with $380 \mathrm{gm}$ load

The elastomer ribbon's actuation properties are elaborated as stress and strain function of the applied voltage as shown in Fig. 6. Besides the quadratic dependency on the applied voltage and the intrinsic non-linear stress-strain characteristic, the actuator performance is increased by the applied prestrain increases for the development of the displacement range and force.

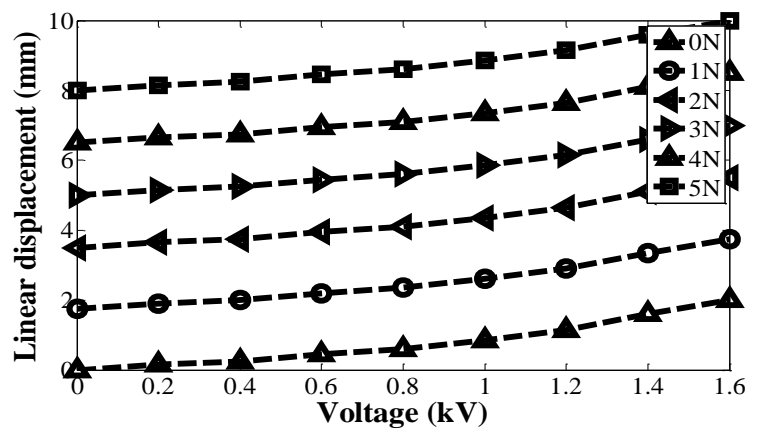

Fig. 6. Voltage-elongation relationships of one of the elastomer actuators.

\section{CONCLUSIONS}

A variety of designs for flexible snake robot actuator while utilizing an EAP model-guided approach to assist in actuator design is analysed in this paper. Though both long, thin samples and wide-strip samples were characterized, it was later determined that only the wide-strip elastomer configuration was practical for use as an actuator to produce force and stroke.
Therefore, most of the subsequent designs for a flexible arm were based on a wide-strip EAP as its main actuator.

Dielectric elastomer EAPs are promising robotics actuators in bio-inspired artificial robot. They show good mechanical performance, are very low weight, simple and low cost. An important characteristic of DEs is that their polymeric nature makes their performance, reliability, and efficiency highly stretch rate dependant. In particular, the best material known to date for DEAs, VHB 4910, shows improved performance and reliability when operating at high stretch rates. Furthermore, An investigation has been made here are for the tendon driven platform. This step requires further developments, but at the very least, what is evident is the viability, to a limited degree of the proposed design. As the guiding priority is to keep the design small and simple, a successful experimental test would certainly be promising. This development will enhance the inspiration of the researchers to produce more lightweight micro scale actuator in future.

\section{REFERENCES}

[1] W. Kier and M.P. Stella, "The arrangement and function of octopus arm musculature and connective tissue", Journal of Morphology, 2007, vol. 268, pp.831-843.

[2] Y. Yekutieli, G. Sumbre, T. Flash, and B. Hochner, "How to move with no rigid skeleton? The octopus has the answers", Biologist, 2002, vol. 49(6), pp. 250-254

[3] I. D. Walker, D. M. Dawson, T. Flash, F. W. Grasso, R. T. Hanlon, B. Hochner, W. M. Kier, C. C. Pagano, C. D. Rahnh, Q. M. Zhang, "Continuum robot arms inspired by cephalopods", Proc. SPIE, 2005, Vol. 5804, pp. 303.

[4] D. Trivedi, C.D. Rahn, W.M. Kier, and I.D. Walker, "Soft robotics: biological inspiration, state of the art, and future research", Advanced Bionics and Biomechanics, September 2008, Vol. 5, No. 2, pp. 99-117.

[5] D. B. Camarillo, C. F. Milne, C. R. Carlson, M. R. Zinn, and J. K. Salisbury, 2008, "Mechanics modeling of tendon-driven continuum manipulators", IEEE Trans. on Robotics, 24(6):1262-1273.

[6] K. J. Cho and H. H. Asada, "Architecture design of a multiaxis cellular actuator array using segmented binary control", IEEE Transactions on Robotics, Vol. 22, 2006, no. 4, pp. 831-843.

[7] P. Lochmatter, "Development of a shell-like electroactive polymer (EAP) actuator", D-MAVT. Zuerich, ETH Zurich. Dr. sc. techn. 2007, pp. 17-21.

[8] M. Wissler, E. Mazza, "Modeling and simulation of dielectric elastomer actuators", Smart Materials and Structures, 2005, No. 14, ISSN. 13961402

[9] G. Kovacs, L. During, "Contractive tension force stack actuator based on soft dielectric EAP", Proc. of SPIE, 2009, Vol. 7287, ISSN. 72870A-1.

[10] F. Carpi, C. Salaris, D. De Rossi, 2007, "Folded dielectric elastomer actuators", Smart Materials and Structures, 2007, No. 16, ISSN. S300S305.

[11] Q. Pei, M. Rosenthal, S. Stanford, H. Prahlad, R. Pelrine, "Multipledegrees-of- freedom electro elastomer roll actuators", Smart Materials and Structures, 2004, No. 13, pp. 86-92.

[12] P. Brochu, Q. Pei, "Advances in dielectric elastomers for actuators and artificial muscles", Macromolecular Rapid Communications, 2010, No. 31 , pp. 10-36.

[13] A. E. Green, W. Zerna, "Theoretical Elasticity", Oxford University Press, 2002, ISBN 0-486-49507-8. CH 3.

[14] G. Kofod, P. Sommer-Larsen, R. Kornbluh, R. Pelrine, "Actuation response of polyacrylate dielectric elastomers", J. Intell. Mater. Syst. Struct., 2003, No. 14, pp. 78-93. 